\title{
GaN light-emitting triodes for high-efficiency hole injection and light emission
}

\author{
Jong Kyu Kim ${ }^{\mathrm{a},{ }^{*},}$ J.-Q. Xi ${ }^{\mathrm{a}}$, Hong Luo ${ }^{\mathrm{a}}$, Jaehee Cho ${ }^{\mathrm{b}}$, Cheolsoo Sone ${ }^{\mathrm{b}}$, Yongjo Park ${ }^{\mathrm{b}}$, Thomas \\ Gessmann $^{\mathrm{a}}$, J. M. Zavada ${ }^{\mathrm{c}}$, H. X. Jiang ${ }^{\mathrm{d}}$, and E. Fred Schubert ${ }^{\mathrm{a}}$ \\ ${ }^{a}$ The Future Chips Constellation, Rensselaer Polytechnic Institute, Troy, NY 12180 \\ ${ }^{b}$ Photonics Program Team, Samsung Advanced Institute of Technology, Suwon 440-600, South \\ Korea \\ ${ }^{\circ}$ US Army Research Office, Research Triangle Park, North Carolina 27709 \\ ${ }^{\mathrm{d}}$ Department of Physics, Kansas State University, Manhattan, Kansas 66506
}

\begin{abstract}
Experimental results on a new type of light-emitting device, the light-emitting triode (LET), are presented. The LET is a three-terminal p-n junction device that accelerates carriers in the lateral direction, i.e. parallel to the p-n junction plane, by means of an electric field between two anodes. The lateral field provides additional energy to carriers thereby allowing them to overcome barriers and increasing the carrier injection efficiency into the active region. LETs were fabricated using a ultraviolet LED structure that has an $\mathrm{AlGaN} / \mathrm{GaN}$ superlattice in the p-type confinement region for high-conductivity 2 dimensional hole gas. LET mesa structures were obtained by standard photolithographic patterning followed by chemically-assisted ion-beam etching using $\mathrm{Cl}_{2}$ and $\mathrm{Ar}$ to expose the n-type cladding layer. The n-type contact was fabricated by electron-beam evaporation of Ti/Al/Ni/Au. Ni/Au $(50 / 50 \AA)$ metallization was deposited for both anodes, Anode 1 and Anode 2, and subsequently annealed at $500{ }^{\circ} \mathrm{C}$ in an $\mathrm{O}_{2}$ ambient. It is shown that both the current between Anode 1 and the cathode, and the light-output power increase with increasing negative bias to the Anode 2. This is consistent with the expectation that a negative bias to the second anode allows carriers to acquire a high kinetic energy thereby enabling them to overcome the barrier for holes, resulting in high injection efficiency into the active region that lies beyond the barrier.
\end{abstract}

Keywords: light-emitting diodes, light-emitting triodes

\section{Introduction}

AlGaN-based ultraviolet (UV) light-emitting diodes (LEDs) are attracting much attention for applications such as chemical and biological detection systems, water and air sterilization, and as primary light source for phosphor-based white LEDs. ${ }^{1 \sim 3}$ Although UV LEDs are already commercially available, highly efficient UV LEDs are still difficult to fabricate. Improvement of the efficiency is one of the most important challenges especially for deep UV LEDs $(\lambda<340$ $\mathrm{nm}$ ) which have very low internal quantum efficiency. In AlGaN-based UV LEDs, an electron-blocking layer (EBL) is frequently inserted between the p-type cladding layer and the active region. The EBL has the purpose of preventing electron overflow from the active region, and hence, confining electrons to the active region. Figure 1 (a) shows a schematic band diagram of a UV LED with an EBL on a multiple quantum well (MQW) active region. The EBL does not impede hole injection into the active region, if the EBL is heavily p-doped. However, AlGaN with high Al content generally lacks high p-type doping capability which is caused by the high acceptor activation energy of $>200 \mathrm{meV}$. If the EBL is undoped or low p-doped, it will not only block electrons from escaping the active region but also hinder the injection of holes into the active region by the potential barrier, as shown in the Fig. 1 (a). The tunneling probability of holes through the EBL is low due to a high potential barrier as well as the heavy mass of holes in $\mathrm{GaN}\left(m_{\mathrm{h}}{ }^{*}=0.80 \times m_{\mathrm{e}}\right)$ and AlN $\left(m_{\mathrm{h}}{ }^{*}=3.53 \times m_{\mathrm{e}}\right)$. This limits the hole injection efficiency into the active region, and hence internal quantum efficiency.

In order to overcome the lack of $p$-type conductivity in bulk $\mathrm{Al}_{x} \mathrm{Ga}_{1-x} \mathrm{~N}$ films, $\mathrm{Mg}$ doped $\mathrm{Al}_{x} \mathrm{Ga}_{1-x} \mathrm{~N} / \mathrm{GaN}$ superlattices (SLs) have been proposed and demonstrated to have a doping efficiency that is 10 times higher than that of bulk p-type $\mathrm{GaN}^{4,5}$ The enhancement of carrier transport has been verified in lateral direction, i. e. parallel to the SLs planes.

*kimj4@rpi.edu; phone 1518276 6151; fax 15182768042

Light-Emitting Diodes: Research, Manufacturing, and Applications X,

edited by Klaus P. Streubel, H. Walter Yao, E. Fred Schubert, Proc. of SPIE Vol. 6134, 61340K, (2006)

0277-786X/06/\$15 - doi: 10.1117/12.647453

Proc. of SPIE Vol. 6134 61340K-1 
However, carrier transport along the perpendicular direction through p-n junction, which is required in typical lightemitting devices, is less efficient than along the lateral direction because the most of the holes ionized from the acceptors are localized inside the quantum wells which are clad by potential barriers as high as 100 to $400 \mathrm{meV}{ }^{6}$ The hindrance of carriers in overcoming the barrier results in a low injection efficiency of carriers in the active region, leading to poor internal quantum efficiency of LEDs. Figure 1 (b) shows a schematic band diagram of an LED with p-type SLs on top of the MQW active region. A schematic description of electron and hole transport in the LED structure is also shown. Efficient injection of holes into the active region of a LED is required for high radiative efficiency. For inefficient hole injection into the active region, electrons will diffuse through the active region into the p-type confinement layer, where non-radiative recombination is likely.
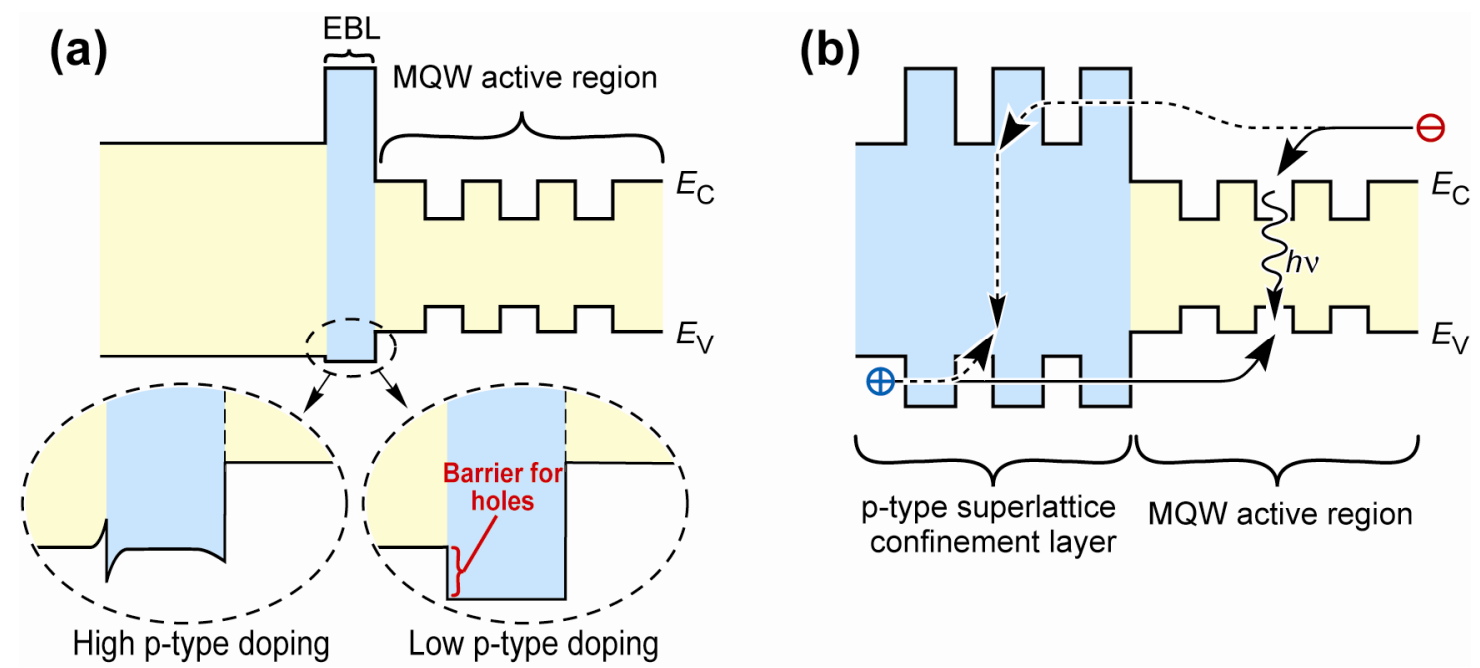

Fig. 1. (a) Schematic band diagram of a UV LED with electron -blocking layer (EBL) on multiple quantum well (MQW) active region. (b) Schematic band diagram of a UV LED showing electron and hole transport in lightemitting diode with p-type superlattice confinement region.

The light-emitting triode (LET) has been motivated by the problem of low injection efficiency in LEDs having a ptype superlattice in the confinement region. ${ }^{7 \sim 9}$ Schematic sketches of the LED and LET are shown in Fig. 2(a) and 2(b), respectively. Radiative recombination in active region is strongly required for high-efficiency light-emitting devices. In LED, however, non-radiative recombination occurs in p-type confinement region due to an inefficient hole injection over the potential barrier, either by EBL or SLs. The LET is similar to a conventional LED with one important difference: The LET has three terminals, two of which are p-type terminals, Anode 1 and Anode 2, and one of which is an n-type terminal, cathode, as shown in Fig 2(b). The fundamental operating principle of the LET is as follows. We assume that the cathode is grounded and the two anodes are both biased at a different level. As a result, a current will flow laterally from one anode to the other anode. In the process, the holes will be accelerated, gain a higher energy, and hence be able to overcome the potential barrier by either EBL or SLs and be injected into the active region more easily as compared to a device with one anode. Thus, the LET structure is a promising new structure with potential advantages for devices that have SLs in the p-type confinement region as well as for UV devices that have an EBL. 


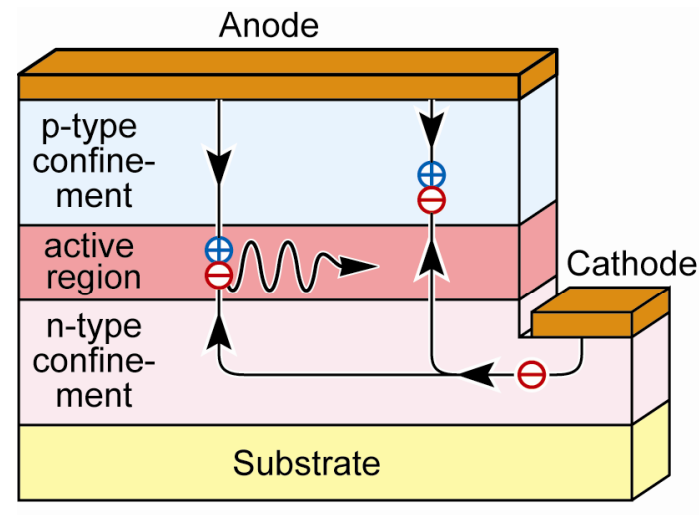

(a) Light-emitting diode (LED)

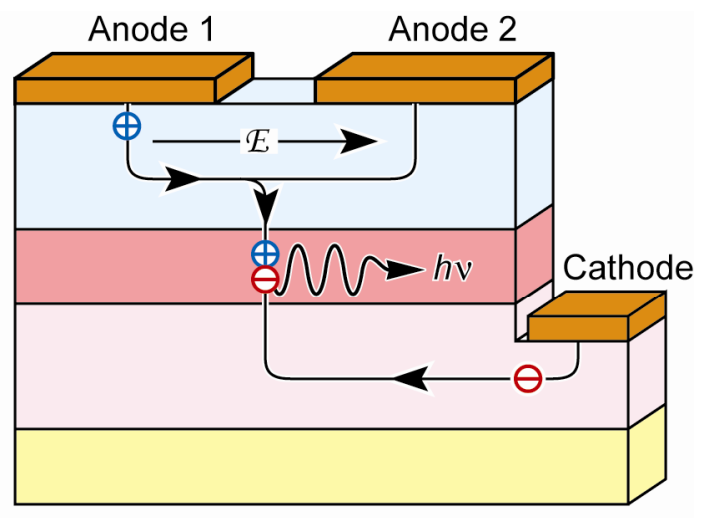

(b) Light-emitting triode (LET)

Fig. 2. (a) Light-emitting diode with non-radiative recombination in p-type confinement region. (b) Lightemitting triode with enhanced hole injection into active region and reduced recombination in p-type confinement layer. Schematic cross-sectional view of metal reflector, distributed Bragg reflector (DBR), and triple-layer omni-directional reflector (ODR).

\section{Theoretical calculation}

The carrier temperature can be obtained by equating the energy-gain rate by an electric field to the energy-loss rate by optical phonon scattering. Under steady-state conditions, the two rates are identical, i.e.

$$
\left.\frac{\mathrm{d} E}{\mathrm{~d} t}\right|_{\text {electric field }}=\left.\frac{\mathrm{d} E}{\mathrm{~d} t}\right|_{\text {phonon scattering }}
$$

with

$$
\left.\frac{\mathrm{d} E}{\mathrm{~d} t}\right|_{\text {electric field }}=e \mathscr{E} v=e \mathscr{E} \mu \mathcal{E}=e \mu \mathcal{E}^{2}
$$

and

$$
\left.\frac{\mathrm{d} E}{\mathrm{~d} t}\right|_{\text {phonon scattering }}=\frac{\frac{3}{2} k\left(T_{c}-T\right)}{\tau_{e}}
$$

where $\mu$ is the carrier mobility, $\mathcal{E}$ is the electric field, $T$ is the lattice temperature, $T_{\mathrm{c}}$ is the carrier temperature, and $\tau_{\mathrm{e}}$ is the optical phonon scattering time. Inserting Eqs. (2) and (3) into Eq. (1) and solving Eq. (1) for the carrier temperature yields

$$
\Delta T_{\mathrm{c}}=T_{\mathrm{c}}-T=e \mu E^{2} \tau_{\mathrm{e}} \frac{2}{3 k}
$$

Using the Boltzmann distribution, one can calculate the ratio of the carrier concentration at the top of the barrier to the carrier concentration at the bottom of the barrier. This ratio is given by

$$
\frac{p_{\text {top }}}{p_{\text {bottom }}}=\exp \left(-\frac{E_{\text {barrier }}}{k T_{\mathrm{c}}}\right)
$$


where the height of the barrier is given by $E_{\text {barrier. }}$. Assuming that the carrier concentration at the bottom of the barrier is constant, the change in carrier concentration at the top is given by

$$
\frac{\left.p_{\text {top }}\right|_{\text {with field }}}{\left.p_{\text {top }}\right|_{\text {without field }}}=\exp \left(\frac{E_{\text {barrier }}}{k T}-\frac{E_{\text {barrier }}}{k T_{\mathrm{c}}}\right) \text {. }
$$

This equation directly gives us the expected improvement in the hole injection into the active region of the LET. Figure 3 shows the change in carrier temperature $\Delta T_{\mathrm{c}}$ and the ratio of carrier concentration at the top of the barrier $p_{\text {with }}$ field $/ p_{\text {without field }}$ as a function of the distance between the two anodes. In the calculation, typical optical phonon scattering time of $\tau_{\mathrm{e}}=10^{-12} \mathrm{~s}$, a p-type GaN hole mobility of $10 \mathrm{~cm}^{2} /(\mathrm{V} \mathrm{s})$, a bias voltage of $5 \mathrm{~V}$, a lattice temperature of $T=300$ $\mathrm{K}$, and a barrier height of $E_{\text {barrier }}=200 \mathrm{meV}$ are used. Both $\Delta T_{\mathrm{c}}$ and $p_{\text {with field }} / p_{\text {without field }}$ increase with decreasing the distance between two anodes, i.e., with increasing electric field.

Note that the $\Delta T_{\mathrm{c}}$ and $p_{\text {with field }} / p_{\text {without field }}$ increase rapidly at electric field higher than $\sim 15 \times 10^{5} \mathrm{~V} / \mathrm{m}$. For example, one obtains a hole-temperature increase of $\Delta T_{\mathrm{c}}=7.7 \mathrm{~K}$, and a corresponding improvement in hole injection by a factor of 1.2 at the distance of $d=5 \mu \mathrm{m}$, which gives an electric field of $10^{6} \mathrm{~V} / \mathrm{m}$. At the distance of $d=1 \mu \mathrm{m}$, i.e. electric field of $5 \times 10^{6} \mathrm{~V} / \mathrm{m}$, the increase of hole-temperature is as high as $\Delta T_{\mathrm{c}}=193 \mathrm{~K}$. This corresponds to an improvement in hole injection over the potential barrier by a factor of 20.3. This is a very encouraging theoretical result that motivates the pursuit of the LET. In addition, this calculation suggests a small lateral dimensions between the two anodes in order to get a high electric field that elevates the hole temperature for a reasonable inter-anode bias range. Scaling down the distance between the Anode 1 and Anode 2 will afford a reduction in lateral bias. LETs with spiral- and interdigitatedtype anodes were designed and fabricated to meet these requirements.

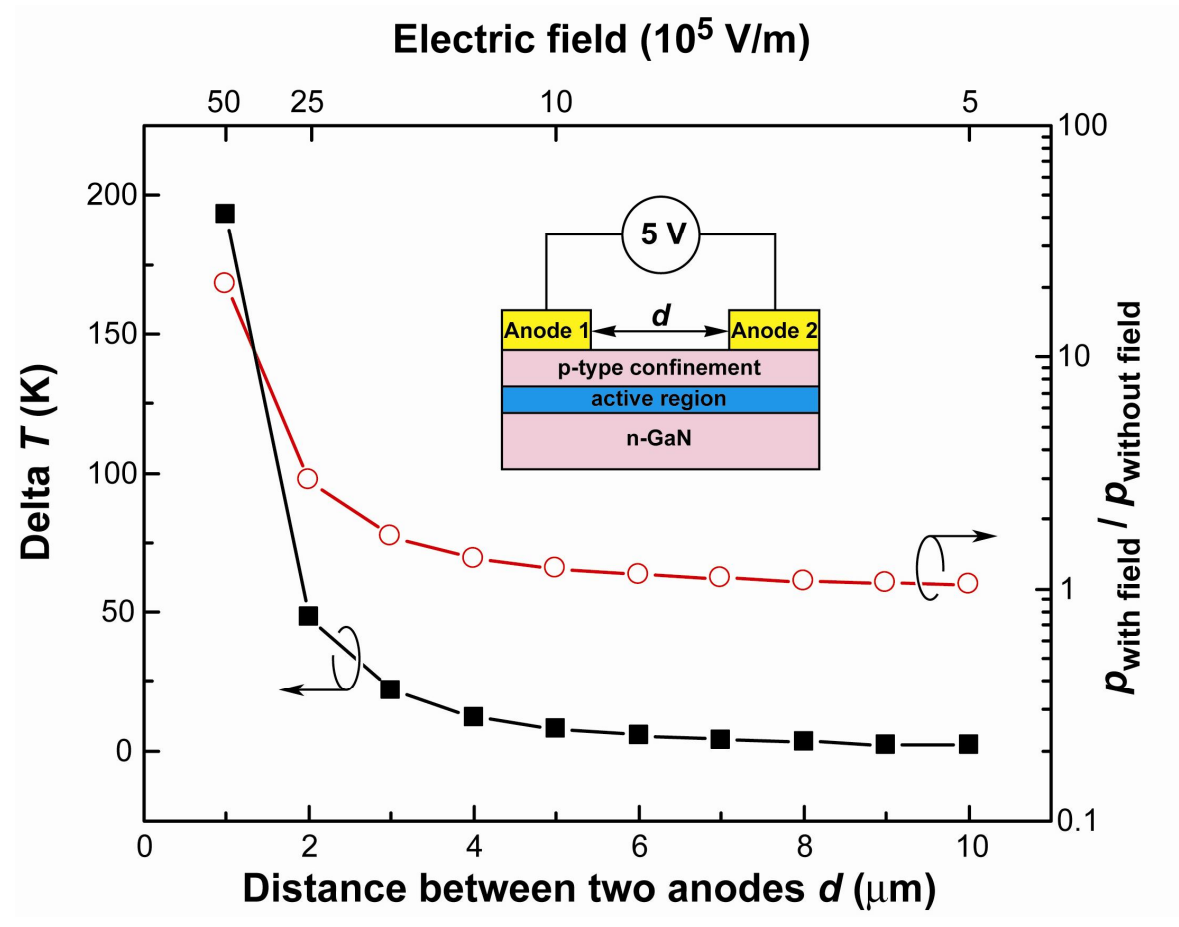

Fig. 3. The variation in carrier temperature $\Delta T_{\mathrm{c}}$ and carrier concentration at the top of the barrier by the bias of $5 \mathrm{~V}$ between the two anodes as a function of the distance between the two anodes.

\section{Experiment and results}

LETs were fabricated on two different UV LED wafers. One is a deep UV LED wafer with peak wavelength of $\lambda_{\text {peak }}$ $=305 \mathrm{~nm}$ having a p-type $\mathrm{Al}_{0.35} \mathrm{Ga}_{0.65} \mathrm{~N}$ EBL . The other is a UV LED wafer with peak wavelength of $\lambda_{\text {peak }}=400 \mathrm{~nm}$ 
having an $\mathrm{Al}_{0.15} \mathrm{Ga}_{0.85} \mathrm{~N} / \mathrm{GaN}$ p-type SL. The $305 \mathrm{~nm}$ UV LED structure was grown by metal-organic chemical vapor deposition (MOCVD) on c-plane sapphire substrate and consists of a $1 \mu \mathrm{m}$-thick AlN buffer layer, a $3 \mu \mathrm{m}$-thick n-type $\mathrm{Al}_{0.2} \mathrm{Ga}_{0.8} \mathrm{~N}$ lower cladding layer, a $\mathrm{Al}_{0.2} \mathrm{In}_{0.03} \mathrm{Ga}_{0.77} \mathrm{~N} / \mathrm{Al}_{0.11} \mathrm{In}_{0.03} \mathrm{Ga}_{0.86} \mathrm{~N}(25 / 25 \AA)$ double quantum well active region, a $20 \mathrm{~nm}$-thick p-type $\mathrm{Al}_{0.35} \mathrm{Ga}_{0.65} \mathrm{~N}$ EBL, and a highly doped p-type GaN contact layer. The $400 \mathrm{~nm}$ UV LED structure was grown by MOCVD on c-plane sapphire substrate and consists of a $2 \mu \mathrm{m}$-thick undoped GaN buffer layer, an $3 \mu \mathrm{m}$-thick n-type GaN lower cladding layer, a 6 period of InGaN/GaN MQW active region, a 20 nm-thick p-type $\mathrm{Al}_{0.15} \mathrm{Ga}_{0.85} \mathrm{~N}$ EBL, a 13 periods of p-type $\mathrm{Al}_{0.15} \mathrm{Ga}_{0.85} \mathrm{~N} / \mathrm{GaN}(25 / 50 \AA) \mathrm{SLs}$, and a highly doped p-type GaN contact layer. A $400 \mathrm{~nm}$ UV LED wafer without p-type SL was also used for LET fabrication in order to investigate the effect of the SLs on hole injection efficiency.

LET mesa structures were obtained by standard photolithographic patterning followed by chemically-assisted ionbeam etching using $\mathrm{Cl}_{2}$ and $\mathrm{Ar}$ to expose the n-type cladding layer. The n-type ohmic contact was fabricated by electronbeam evaporation of $\mathrm{Ti} / \mathrm{Al} / \mathrm{Ni} / \mathrm{Au}$ and annealing at $650^{\circ} \mathrm{C}$ for 1 min in $\mathrm{N}_{2}$ ambient. $\mathrm{Ni} / \mathrm{Au}(5 / 5 \mathrm{~nm})$ ohmic contact layer to p-type GaN was deposited and subsequently annealed at $500{ }^{\circ} \mathrm{C}$ in an $\mathrm{O}_{2}$ ambient. Finally, bonding metal was deposited by electron-beam evaporation of $\mathrm{Cr} / \mathrm{Au}(20 / 500 \mathrm{~nm})$. Figure 4 shows optical micrographs of spiral- and interdigitated-type LETs. The chip dimension is $1 \times 1 \mathrm{~mm}^{2}$ for the spiral-type LET, and $300 \times 300 \mu \mathrm{m}^{2}$ for the interdigitated-type LET. The distance between two anodes is $10 \mu \mathrm{m}$ for the spiral-type LET, and $4 \mu \mathrm{m}$ for the interdigitated-type LET.
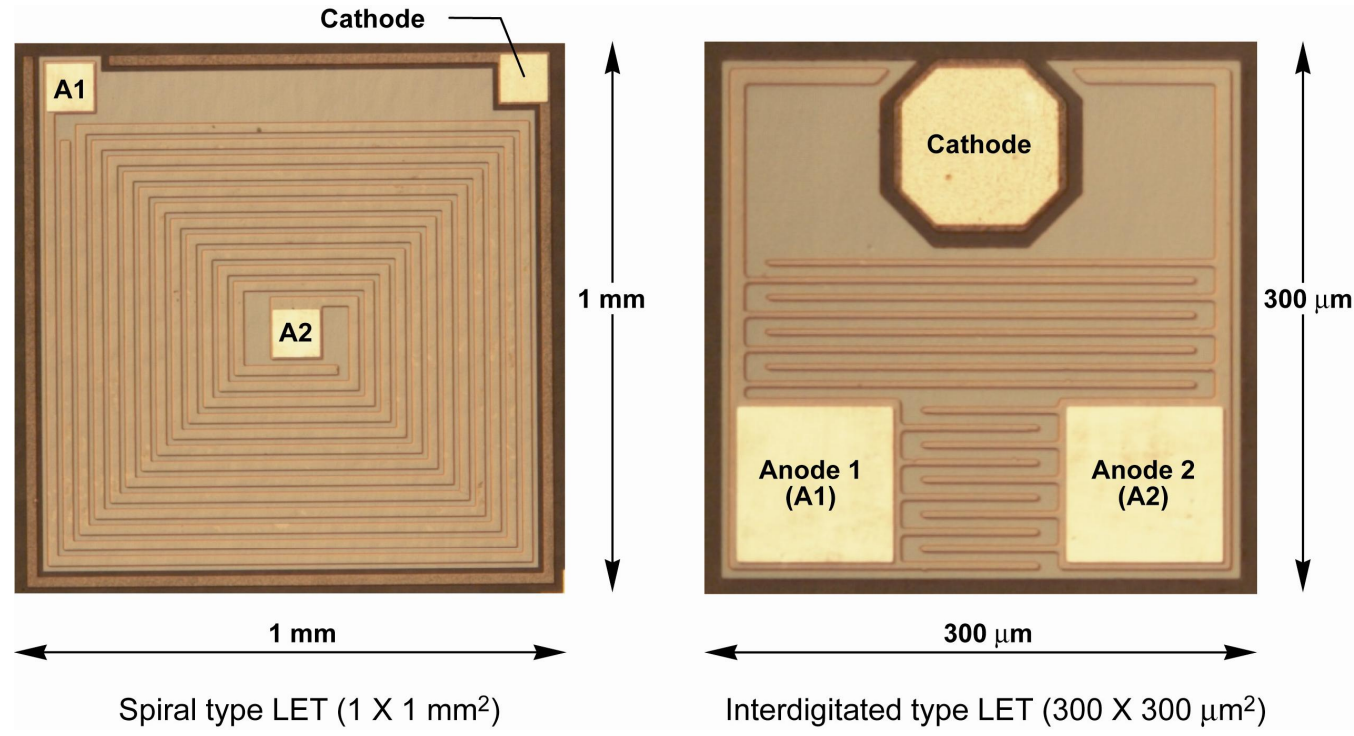

Fig. 4. Optical micrographs of spiral- and interdigitated-type LETs

The electrical properties of the $305 \mathrm{~nm}$ UV LETs are shown in Fig. 5. As a negative bias to Anode 2, $V_{\mathrm{G}}$, increases, the current between Anode 1 and cathode increases, as shown in Fig. 5(a). Fig. 5 (b) shows the transconductance, $\mathrm{G}_{\mathrm{m}}=$ $\left[\partial I_{\mathrm{Al}-\mathrm{C}} / \partial V_{\mathrm{G}}\right]_{\mathrm{VF}=\mathrm{contant}}$, for different voltages between Anode 1 and cathode, $V_{\mathrm{F}}$. As the $V_{\mathrm{G}}$ increases negatively, $G_{\mathrm{m}}$ becomes more negative, which is attributed to an enhanced hole injection efficiency over the potential barrier by the $\mathrm{Al}_{0.35} \mathrm{Ga}_{0.65} \mathrm{~N}$ EBL. 

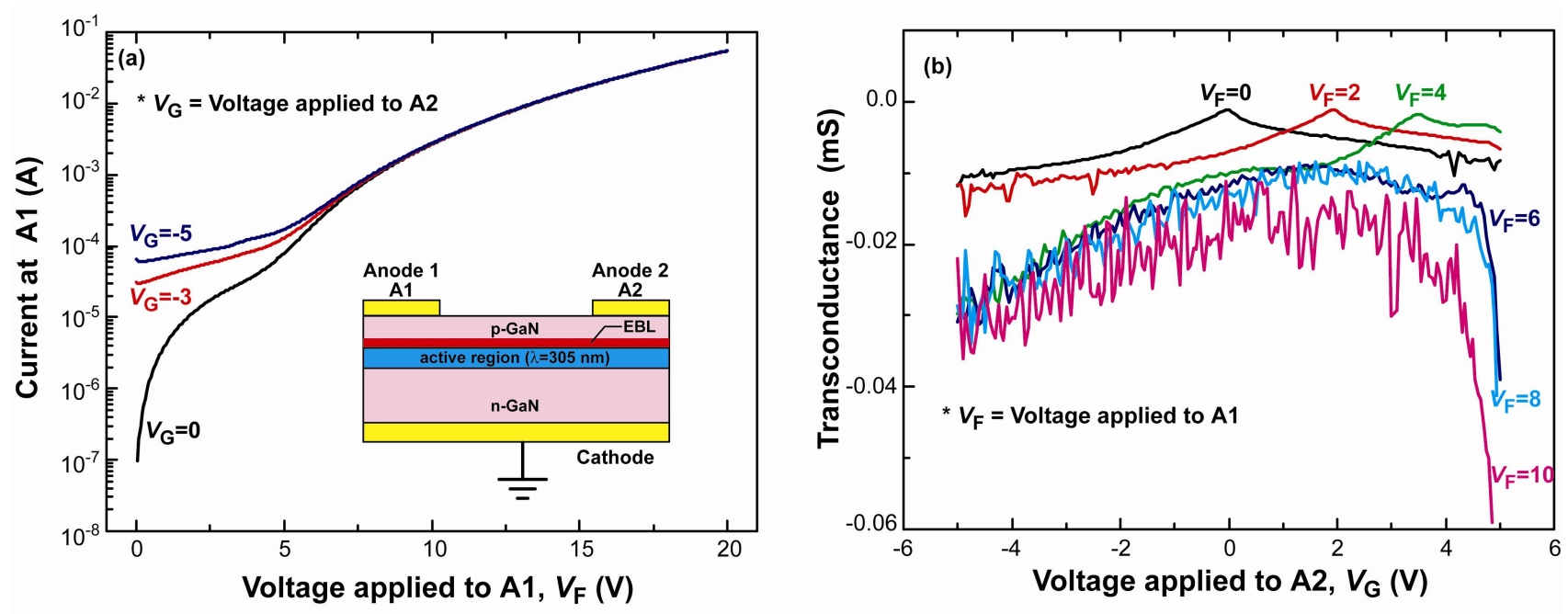

Fig. 5. (a) Current-voltage characteristics and (b) transconductance, $G_{\mathrm{m}}=\left[\partial I_{\mathrm{A} 1-\mathrm{C}} / \partial V_{\mathrm{G}}\right]_{\mathrm{VF}=\text { contant }}$, of the $305 \mathrm{~nm}$ UV LET.

The electroluminescence intensity from the backsides of the LETs was measured directly on a large-size $(10 \times 10$ $\mathrm{mm}^{2}$ ) Si PIN photodetector. The light-output-versus-current characteristic of the $305 \mathrm{~nm}$ UV LETs is shown in Fig. 6. Inspection of the figure clearly reveals that the light output power depends on the bias to Anode $2, V_{\mathrm{G}}$, particularly at small forward current values. As the $V_{\mathrm{G}}$ decreases, both the current through cathode and the light-output power increase. This is consistent with the theoretical consideration that a negative bias to the second anode allows carriers to acquire a high kinetic energy thereby enabling them to overcome the barrier for holes, resulting in high hole injection efficiency into the active region that lies beyond the barrier. Although this result was obtained for a UV LET structure with an EBL, it should be reproducible for LET structures employing SLs for the p-type cladding layer.

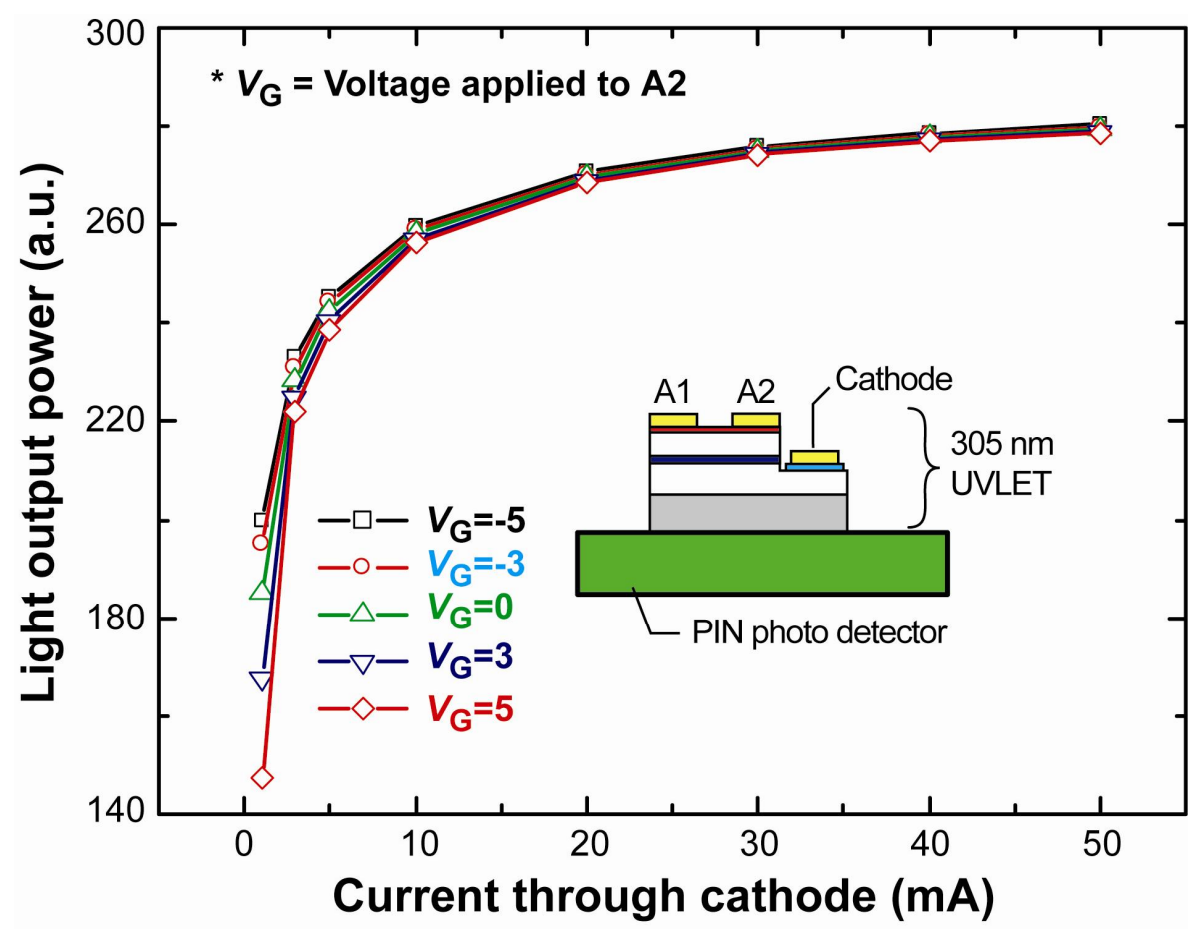

Fig. 6. (light-output power-versus-current characteristic of the $305 \mathrm{~nm}$ UV LETs. The lightoutput power from the backside of the LET was measured directly on Si PIN photodetector. 
The light-output-versus-current characteristics of the $400 \mathrm{~nm}$ UV LETs without SLs and with AlGaN/GaN SLs are shown in Fig. 7 (a) and (b), respectively. No change in light-output with $V_{\mathrm{G}}$ is observed for $400 \mathrm{~nm}$ UV LET without SLs. However, for the $400 \mathrm{~nm}$ UV LET with AlGaN/GaN SLs, the light output power depends on the bias to Anode 2, $V_{\mathrm{G}}$. As the $V_{\mathrm{G}}$ decreases, the light output power increases even though the current through cathode remains constant. This is an important finding and consistent with the expectation that UV LET structure employing SLs as well as employing an EBL showed enhanced light output with increasing negative bias to the additional anode A2. A negative bias to the second anode allows carriers to acquire a high kinetic energy thereby enabling them to overcome the barrier for holes, resulting in high injection efficiency into the active region that lies beyond the barrier. Furthermore, it is worthwhile to note that there is no ambiguity at all with respect to the current that flows through the pn junction. The current measured at the n-type contact must necessarily flow through the pn junction. Therefore, the results unambiguously confirm that the validity of the LET principle.
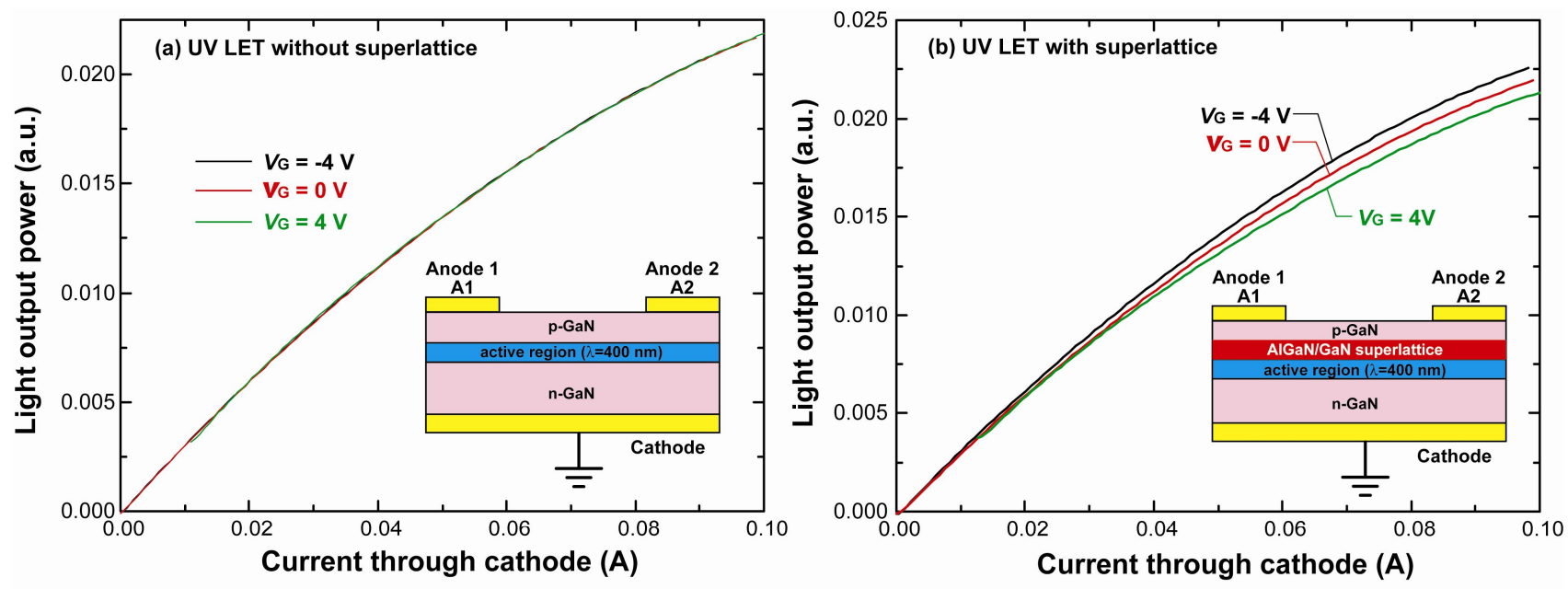

Fig. 7. The light-output power-versus-current characteristic of the $400 \mathrm{~nm}$ UV LETs (a) without and (b) with AlGaN/GaN SLs.

\section{Conclusion}

In conclusion, a new type of light-emitting device, the light-emitting triode (LET) is demonstrated to have enhanced hole injection efficiency. Theoretical calculations reveals that the lateral electric field of $5 \times 10^{6} \mathrm{~V} / \mathrm{m}$ provides an additional energy to holes increasing their temperature by $\Delta T_{\mathrm{c}}=193 \mathrm{~K}$, thereby allowing them to overcome potential barriers by either superlattices or electron-blocking layer. This leads to the improvement of hole injection efficiency into the active region by a factor of 20.3. UV LETs $\left(\lambda_{\text {peak }}=305 \mathrm{~nm}\right)$ having p-type $\mathrm{Al}_{0.35} \mathrm{Ga}_{0.65} \mathrm{~N}$ EBL and UV LETs $\left(\lambda_{\text {peak }}=\right.$ $400 \mathrm{~nm}$ ) having AlGaN/GaN SLs are fabricated. It is experimentally shown that the light-output power of LETs increases with increasing negative bias to the additional anode. This is consistent with the theoretical consideration that a negative bias to the second anode allows carriers to acquire a high kinetic energy thereby enabling them to overcome the barrier for holes, resulting in high hole injection efficiency into the active region that lies beyond the barrier.

\section{Acknowledgement}

We gratefully acknowledge support from the Army Research Office (ARO), National Science Foundation (NSF), Defense Advanced Research Projects Agency (DARPA), Crystal IS Corporation, and Samsung Advanced Institute of Technology (SAIT). 


\section{Reference}

1. T. Nishida, N. Kobayashi, T. Ban, "GaN-free transparent ultraviolet light-emitting diodes," Appl. Phys. Lett. 82, 1 (2003).

2. S. Shakya, K. H. Kim, H. X. Jiang, "Enhanced light extraction in III-nitride ultraviolet photonic crystal lightemitting diodes," Appl. Phys. Lett. 85, 142 (2004).

3. J. P. Zhang, X. Hu, Y. Bilenko, J. Deng, A. Lunev, M. S. Shur, R. Gaska, M. Shatalov, J. W. Yang, M. A. Khan, "AlGaN-based $280 \mathrm{~nm}$ light-emitting diodes with continuous-wave power exceeding $1 \mathrm{~mW}$ at $25 \mathrm{~mA}$," Appl. Phys. Lett. 85, 5532 (2004).

4. E. F. Schubert, W. Grieshaber, and I. D. Goepfert, "Enhancement of deep acceptor activation in semiconductors by superlattice doping,” Appl. Phys. Lett. 69, 3737 (1996).

5. J. K. Kim, E. L. Waldron, Y.-L. Li, T. Gessmann, E. F. Schubert, H. W. Jang, J.-L. Lee, "P-type conductivity in bulk $\mathrm{Al}_{x} \mathrm{Ga}_{1-x} \mathrm{~N}$ and $\mathrm{Al}_{x} \mathrm{Ga}_{1-x} \mathrm{~N} / \mathrm{Al}_{y} \mathrm{Ga}_{1-y} \mathrm{~N}$ superlattices with average Al mole fraction $>20 \%$," Appl. Phys. Lett. 84, 3310 (2004).

6. E. L. Waldon, Y.-L. Li, E. F. Schubert, J. W. Graff, J. K. Sheu, "Experimental study of perpendicular transport in weakly coupled $\mathrm{Al}_{x} \mathrm{Ga}_{1-x} \mathrm{~N} / \mathrm{GaN}$ superlattices,” Appl. Phys. Lett. 83, 4975 (2003).

7. S. M. Komirenko, K.W. Kim, V. A. Kochelap, and J. M. Zavada, "Enhancement of hole injection for nitridebased light-emitting devices," Solid-State Electronics 47, 169 (2003).

8. S. Luryi, "Light-emitting devices based on the real-space transfer of hot electrons," Appl. Phys. Lett. 58, 1727 (1991).

9. S. M. Komirenko, K. W. Kim, V. A. Kochelap, J. M. Zavada, "Laterally doped heterostructures for III-N lasing devices,“ Appl. Phys. Lett. 81, 4616 (2002) 